we found
that the
cause of this
phenomenon
was the
buckling of
the liquid
crystalline
domains

PHOTONICS

\section{Crosslinking for colour}

Hydroxypropyl cellulose (HPC) is an eco-friendly polymer which is commonly used in the food and pharmaceutical industries. Once in water the polymer forms a chiral nematic liquid crystalline phase which is structurally coloured, meaning that the colours come from the twisting of the polymer chains, rather than from an absorption mechanism. These colours are bright, iridescent and do not fade over time. However, HPC only reflects visible light when dissolved in water. With air exposure, the iridescent, colourful

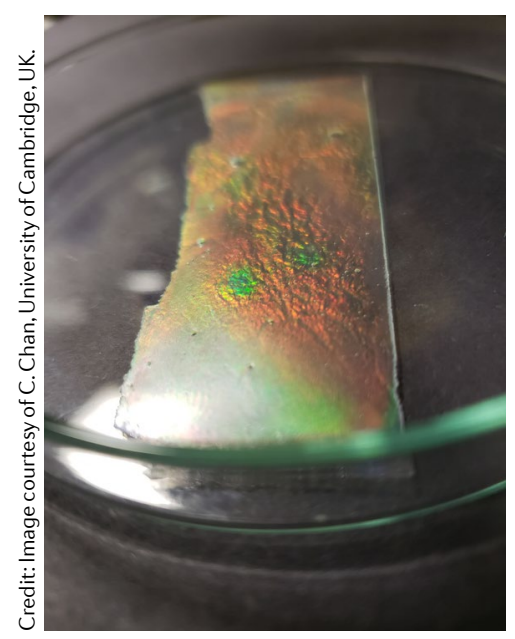

solution dries to give a solid film reflecting only UV light. Retaining the colouration in the solid form is desirable to obtain a colourful coating composed of a renewable and degradable biopolymer. Writing in Advanced Materials, Silvia Vignolini and co-workers now show that chemically-crosslinking HPC enables the colour to be retained in the dried solid coatings.

"We found that using a chemical crosslinker not only allowed to maintain the colour [in the solid films], but small variations of the crosslinker concentration allowed us to access films that reflected across the full spectrum of visible colour," says Clement Chan, first author of the study. By changing the crosslinker concentration from 0 to $5.5 \mathrm{wt} \%$, the team were able to tune the colour of the dry films from violet to infrared.

Surprisingly, the colour of the dry, crosslinked HPC film is independent of the angle or polarization of the incident light. "When the material was examined closely via optical and scanning electron microscopy, we found that the cause of this phenomenon was the buckling of the liquid crystalline domains," explains Vignolini. Drying usually compresses a structure and this buckling was unexpected.

Many factors affect the process of drying an HPC solution into a film, including temperature, mesophase composition, type of bonds and viscosity of the solution. Crosslinking the films caused the mesophase to become more disordered and form multiple domains. When the film dried, there was an effective strain on the material, causing these domains to buckle. This buckling meant that regardless of the angle of incidence, some domains would always be aligned favourably to reflect incoming light.

"Going forward, we are examining how this crosslinking approach can be applied in different geometries, and if the interesting optical properties described in this work manifest beyond simple films," says Vignolini. Understanding the origins of these properties is not only interesting from a fundamental point of view, but also offers a pathway to developing HPC-derived photonic pigments which possess other properties, such as elastic mechanical properties and stimuli responsive behaviour.

Ankita Anirban

ORIGINAL ARTICLE Chan et al. Visual appearance of chiral nematic cellulose-based photonic films: angular and polarization independent color response with a twist. Adv. Mater. https://doi.org/10.1002/adma.201905151 (2019) 